\title{
Utilization of HIV-1 envelope V3 to identify X4- and R5-specific Tat and LTR sequence signatures
}

\author{
Gregory C. Antell 1,2,3, Will Dampier ${ }^{1,2,3}$, Benjamas Aiamkitsumrit ${ }^{1,2}$, Michael R. Nonnemacher ${ }^{1,2}$, \\ Jeffrey M. Jacobson 1,4,5, Vanessa Pirrone ${ }^{1,2}$, Wen Zhong 1,2, Katherine Kercher ${ }^{1,2}$, Shendra Passic 1,2, \\ Jean W. Williams ${ }^{1,2}$, Gregory Schwartz ${ }^{3}$, Uri Hershberg ${ }^{1,3}$, Fred C. Krebs ${ }^{1,2}$ and Brian Wigdahl1,2,6*
}

\begin{abstract}
Background: HIV-1 entry is a receptor-mediated process directed by the interaction of the viral envelope with the host cell CD4 molecule and one of two co-receptors, CCR5 or CXCR4. The amino acid sequence of the third variable (V3) loop of the HIV-1 envelope is highly predictive of co-receptor utilization preference during entry, and machine learning predictive algorithms have been developed to characterize sequences as CCR5-utilizing (R5) or CXCR4utilizing (X4). It was hypothesized that while the V3 loop is predominantly responsible for determining co-receptor binding, additional components of the HIV-1 genome may contribute to overall viral tropism and display sequence signatures associated with co-receptor utilization.

Results: The accessory protein Tat and the HIV-1 long terminal repeat (LTR) were analyzed with respect to genetic diversity and compared by Jensen-Shannon divergence which resulted in a correlation with both mean genetic diversity as well as the absolute difference in genetic diversity between R5- and X4-genome specific trends. As expected, the V3 domain of the gp120 protein was enriched with statistically divergent positions. Statistically divergent positions were also identified in Tat amino acid sequences within the transactivation and TAR-binding domains, and in nucleotide positions throughout the LTR. We further analyzed LTR sequences for putative transcription factor binding sites using the JASPAR transcription factor binding profile database and found several putative differences in transcription factor binding sites between R5 and X4 HIV-1 genomes, specifically identifying the C/EBP sites I and II, and Sp site III to differ with respect to sequence configuration for R5 and X4 LTRs.
\end{abstract}

Conclusion: These observations support the hypothesis that co-receptor utilization coincides with specific genetic signatures in HIV-1 Tat and the LTR, likely due to differing transcriptional regulatory mechanisms and selective pressures applied within specific cellular targets during the course of productive HIV-1 infection.

Keywords: HIV-1, Co-receptor, Tropism, LTR, Tat, V3, gp120, Diversity, Divergence, Transcription factor

\section{Background}

HIV-1 entry is a receptor-mediated, pH-independent process occurring via the direct interaction between viral envelope glycoprotein (gp)120 and the host cell CD4 receptor molecule, as well as one of the two most

\footnotetext{
*Correspondence: bwigdahl@drexelmed.edu

${ }^{1}$ Department of Microbiology and Immunology, Drexel University College of Medicine, Philadelphia, PA, USA

Full list of author information is available at the end of the article
}

commonly encountered co-receptor molecules, CCR5 or CXCR4 [1]. The HIV-1 gp120 entry protein consists of five variable regions (V1-V5), which are highly modified by insertion, deletion, and substitution mutations, interspersed among five constant regions (C1-C5). Regardless of this sequence hypervariability, the overall structure and function of gp120 is highly conserved. Of particular importance to the HIV-1 entry mechanism is the third variable loop (V3), which has been shown to consist of 34-36 amino acid residues (most commonly 
35 residues). The V3 domain has been extensively studied as it has been identified as the principal neutralizing domain (PND) on the viral envelope gp120 [2-4] and the viral partner in selective interactions with the different co-receptors [5].

The V3 region is a major determinant in predicting HIV-1 entry phenotype, distinguishing non-syncytium inducing (NSI) from syncytium inducing (SI) strains and macrophage tropic from non-macrophage tropic strains [5-8]. In this regard, it was discovered that a $\mathrm{T}$ cell tropic (T-tropic) isolate, or SI virus, preferentially uses the coreceptor CXCR4, and has consequently been referred to as an $\mathrm{X} 4$ virus. In contrast, a primary macrophage tropic (M-tropic) isolate, or NSI virus, was found to preferentially use the co-receptor CCR5 for entry and therefore referred to as an R5 virus $[9,10]$. Previously, viral tropism based on co-receptor usage was used interchangeably with tropism defined by cellular target; however, several studies have shown that while co-receptor usage can at times be linked with cellular tropism, it is important to discuss viral infection in terms of utilization of a coreceptor in conjunction with the phenotype of the target cell $[11,12]$. As an example, recent investigations have shown that HIV-1 infectious molecular clones containing transmitted/founder ( $\mathrm{T} / \mathrm{F}$ ) genome sequences preferentially utilized CCR5 as a co-receptor during entry and were able to replicate efficiently in primary CD4+ T cells $[13,14]$. These molecular clones also exhibited reduced replication efficiency in monocyte-derived macrophages (MDMs), in contrast to the prototypic M-tropic strains of HIV-1 [13].

For high-throughput applications, co-receptor utilization predictions can be performed on Env-V3 sequences computationally $[15,16]$. To this end, the internet-based bioinformatic method, position-specific scoring matrices (Web-PSSM), utilizes sequences of known entry phenotype to determine if an Env-V3 sequence is CCR5- or CXCR4-utilizing (R5 or X4 Env-V3 sequences). This algorithm indicates the propensity of the virus to utilize CXCR4 at both high sensitivity (84\%) and specificity (96\%), with X4 virus sequences exhibiting high scores and R5 sequences exhibiting low scores, while intermediate Web-PSSM scores indicates both R5 and X4 virus sequences as well as dual tropic X4/R5 virus sequences [17]. Typically, $\mathrm{X} 4$ viruses emerge gradually in a subset of patients due to accumulation of amino acid changes within the V3 loop, particularly at positions 11 and 25 . While HIV-1 co-receptor usage has been demonstrated to be directly associated with the genotype of V3 loop, as described above, other amino acid residues within gp120 V1, V2, C4, and a number of regions of gp41, have also been associated with co-receptor usage [18-27]. As conformational changes within the V3 loop occur upon the binding of gp120 and CD4, it is possible that the coreceptor usage-associated amino acid residues within other regions of envelope participate in the structural rearrangement of gp120 [28].

The overall goal of this study was to identify and characterize genetic differences between CCR5- and CXCR4-utilizing HIV-1 sequences beyond the V3 loop of envelope as defined by genotypic prediction. Specifically, HIV-1 gp120 and Tat amino acid sequences and the HIV-1 long terminal repeat (LTR) nucleotide sequences were selected for this analysis. The HIV-1 Tat protein derives its name from the fact that its primary function during viral replication is to serve as the trans-activator of transcription. While not directly involved in HIV-1 entry, Tat has a multitude of intracellular host binding partners and functions. The HIV-1 LTR contains an abundance of transcription factor binding sites upstream of the transcription start site that alter levels of HIV-1 transcription, often in a cell type-dependent manner. Importantly, these binding sites can work independently, in concert, or antagonistically, with a single nucleotide polymorphism capable of producing dramatic changes in binding, including the complete abrogation of transcription factor binding $[29,30]$.

We hypothesized that co-evolved R5- or X4-associated genetic signatures emerge in viral genes and proteins that are not directly associated with entry, and suggest that these differences are reflective of evolutionary constraints applied by different cellular milieus that associate, coevolve, or co-adapt with co-receptor usage and may collectively guide tissue- and/or cell type-specific replication patterns, as well as organ-specific disease pathogenesis. The validity of this hypothesis is supported by the association of X4 virus with depleted CD4+ T-cell levels and enhanced disease progression, as well as the tendency of R5 virus to infect cells of the monocyte-macrophage lineage and to be found at the time of transmission [31]. However, the full extent of the underlying changes in the viral genome that could produce such a shift remains unknown.

To remedy this, we have taken a genetic approach with functional underpinnings that have centered on dividing HIV-1 co-linear sequences (gp120, Tat, and LTR) into two co-receptor utilization groups using genotypic prediction methods. Subsequently, we used these two groups of sequences to explore the differences in the remainder of gp120 sequences outside of Env-V3 as well as co-linear Tat and LTR sequences (Table 1). Sequence alignments of each co-receptor usage-defined population of co-linear gp120, Tat, or LTR sequences were quantitatively evaluated at each amino acid (gp120 and Tat) or nucleotide (LTR) position utilizing first-order diversity and Jensen-Shannon divergence. Together, diversity and 
Table 1 Identification of HIV-1 Tat and LTR sequences colinear to CCR5- and CXCR4-utilizing Env-V3 sequences defined by Web-PSSM scoring

\begin{tabular}{lcc}
\hline Genomic region & CCR5 & CXCR4 \\
\hline gp120 & 1678 & 52 \\
Tat & 504 & 31 \\
LTR & 615 & 35 \\
\hline
\end{tabular}

HIV-1 amino acid sequences for gp120 and Tat and nucleotide sequences for LTR were classified as being derived from CCR5- or CXCR4-utilizing genomes according to Web-PSSM prediction scores utilizing the Env-V3 sequence. Sequences with intermediate scores PSSM scores are derived from R5, X4, or dual-tropic X4/R5 viruses and were not included in further analysis

divergence provide metrics to characterize the positionspecific variation of amino acids or nucleotides, as well as provide a quantitative method to compare this information between R5- and X4-defined sequences. This strategy has provided a straightforward genetic approach to define specific sequences in Tat and the LTR, or potentially any other HIV-1 sequence, that are co-selected with CXCR4- and CCR5-dependent entry.

\section{Results and discussion}

Given the goal of this research was to determine if genetic signatures co-evolved between different regions of the HIV genome, it was first necessary to find patients that had sequences from the genomic areas of interest. From the Los Alamos National Library (LANL) database, subtype B sequences derived from patient samples were downloaded and aligned to the HXB2 genome. In total, more than 2500 samples were isolated, which included a full V3 of 35 amino-acids and at least one other co-linear sequence in the Tat or LTR regions. This also included 1730 full gp120 sequences. Table 1 shows the breakdown of sequences for each region and the selection criteria are further described in the Methods. For all results discussed below, we have analyzed the diversity/divergence of the amino acid sequences of gp120 and Tat proteins while the nucleotides of the LTR have been analyzed.

\section{Genetic diversity is correlated in R5- and X4-classified HIV-1 gp120, Tat, and LTR sequence populations}

Spearman's rank correlation was performed to assess the correlation between $\mathrm{R} 5$ and $\mathrm{X} 4$ diversity for $\operatorname{gp} 120\left(\rho=0.8678, P=2.00 \times 10^{-156}\right)$, Tat $\left(\rho=0.8873, P=4.67 \times 10^{-35}\right)$, and LTR $(\rho=0.7021$, $\left.\mathrm{P}=4.06 \times 10^{-78}\right)$ (Fig. 1). In all cases, $\mathrm{R} 5$ and X4 diversity were well-correlated, with the $\mathrm{P}$ value indicating support for the alternative hypothesis that X4 and R5 diversity is unrelated. Because first-order diversity was utilized in this analysis rather than richness (order $=0$ ), and further supported by rarefaction analysis of the sample sizes, it

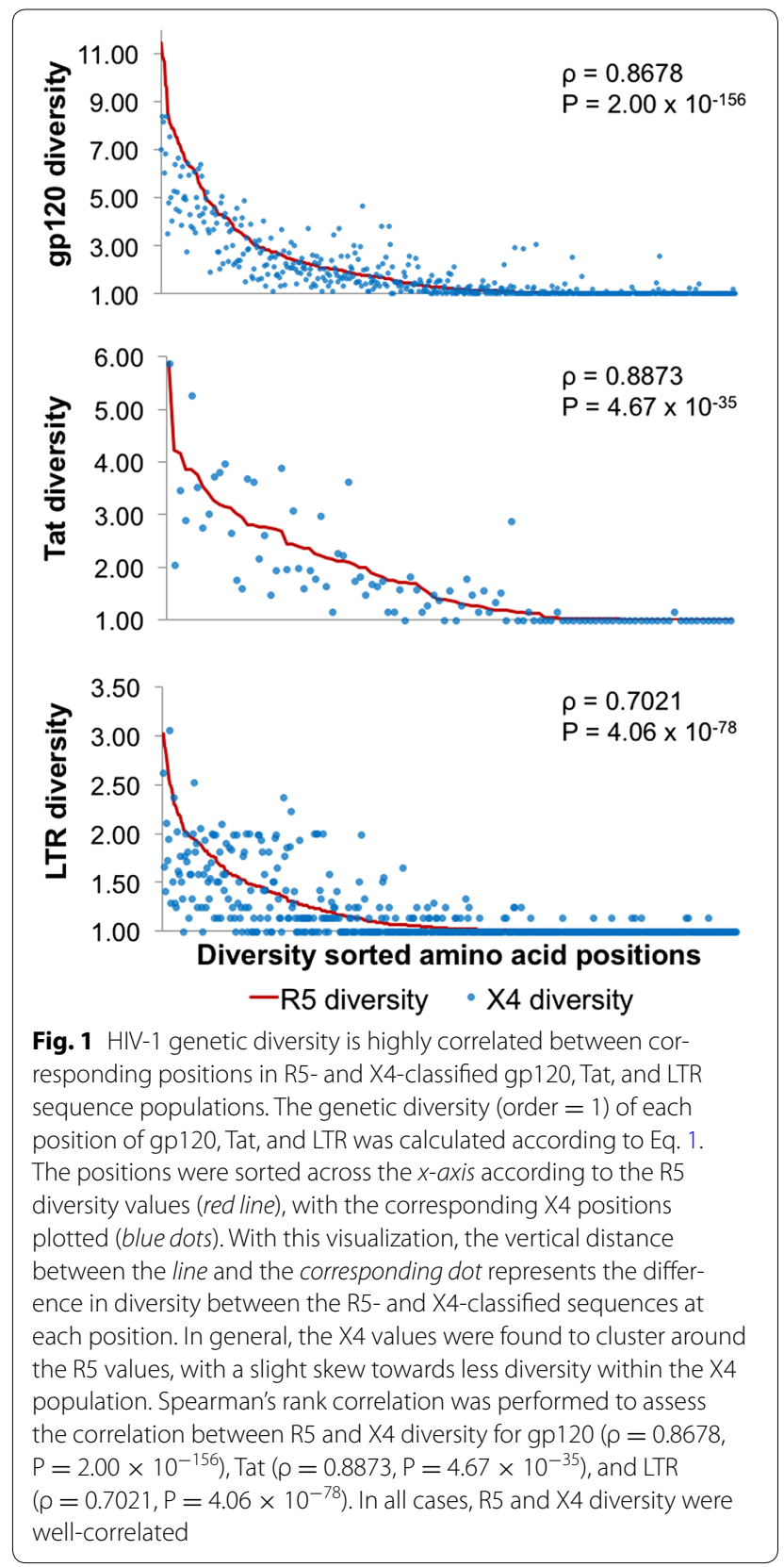

is unlikely that differences in diversity are a reflection of the differences in sample size between the R5 and X4 sequence groups. This result indicates that, in general, corresponding amino acid (gp120 and Tat) or nucleotide (LTR) positions are similarly constrained in their usage with respect to $\mathrm{R} 5$ and $\mathrm{X} 4$ sequences.

\section{Jensen-Shannon divergence correlates with differences in diversity in HIV-1 gp120, Tat, and LTR sequences} Notably, Jensen-Shannon divergence correlates well with both mean diversity ( $\rho=0.9226,0.8552$, and 0.9295 for 
gp120, Tat, and LTR, respectively) and the absolute difference in diversity $(\rho=0.9005,0.8852$, and 0.9685 for gp120, Tat, and LTR, respectively) (Fig. 2). Together, these observations indicate that the gain or loss of diversity in one of the populations is closely associated with high Jensen-Shannon divergence.

\section{Amino acid diversity and Jensen-Shannon divergence identified domains in gp 120 responsible for CCR5 or CXCR4 co-receptor utilization}

The HIV-1 envelope protein gp120 was evaluated to detect the effectiveness of first-order sequence position diversity and Jensen-Shannon divergence with respect to identifying genetic signatures of co-receptor utilization, with the expectation that the V3 domain exhibits higher Jensen-Shannon divergence between R5- and X4-associated gp120 sequences. Diversity analysis, which as stated above was highly correlated between R5 (red) and X4 (blue) sequence populations, confirmed that the variable loops of gp120 display the greatest amount of amino acid diversity (Fig. 3a). The V1 and V4 variable domains, in particular, contain several positions that display diversity of greater than 10 at order $=1$, with a large proportion of positions having a diversity $>3$. Calculation
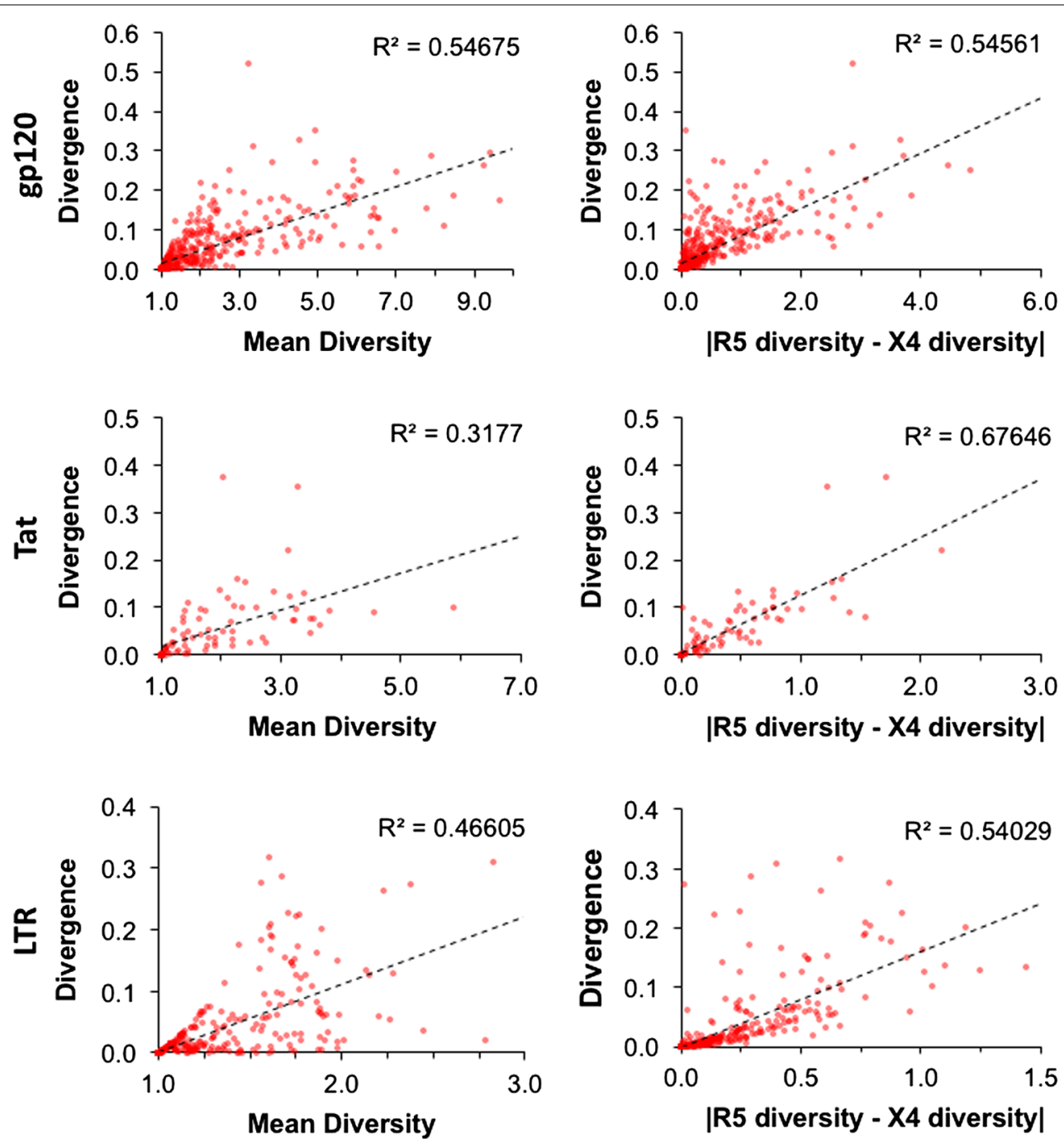

Fig. 2 Jensen-Shannon divergence is correlated with both mean genetic diversity and the absolute difference in genetic diversity. The relationship between Jensen-Shannon divergence and genetic diversity (order = 1) in HIV-1 gp120, Tat, and LTR sequences was evaluated using Spearman's rank correlation. Both the mean diversity of R5- and X4-classified sequences and the absolute difference between R5 and X4 diversity correlated with Jensen-Shannon divergence. This result indicates that large divergence can be a reflection of not only increased amounts of information (as indicated by high mean diversity), but also by the loss of information in one of the two groups (as indicated by the absolute difference in mean diversity) 


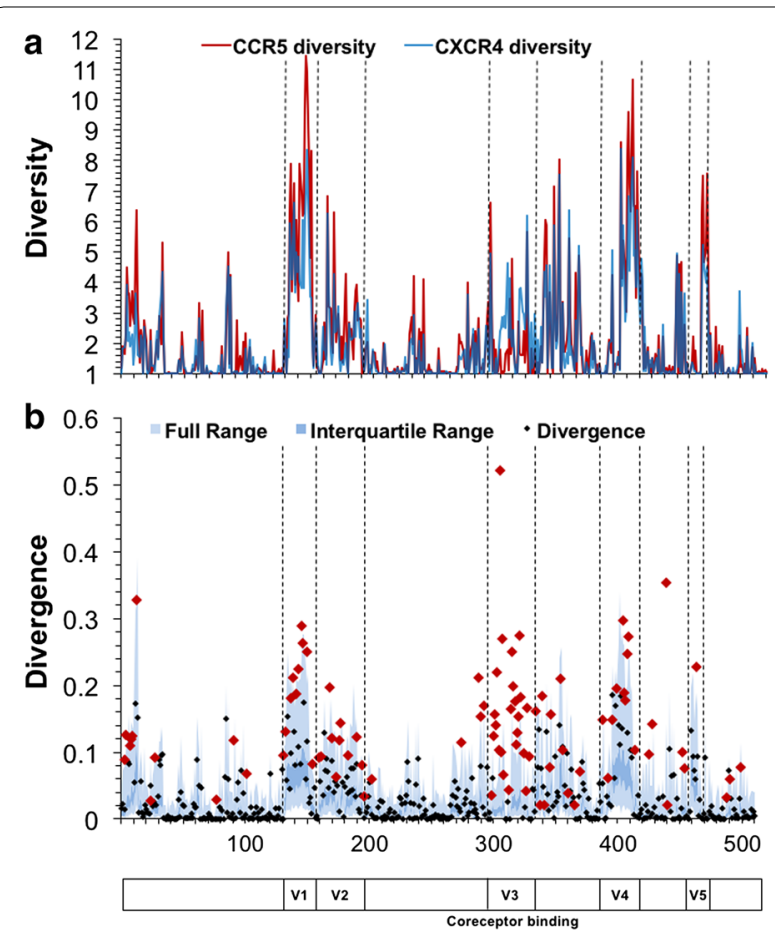

Fig. 3 HIV-1 gp120 demonstrates high Jensen-Shannon divergence in regions with high genetic diversity. HIV-1 gp 120 sequences were classified as CCR5 (R5) ( $n=1681)$ or CXCR4 (X4) ( $n=52)$ according to the predicted co-receptor usage of the $\mathrm{V} 3$ domain Web-PSSM score [17]. a The diversity index at a Hill number of 1 was calculated for each position for both R5 (red) and X4 (blue) gp120 amino acid sequence populations. Diversity values range from 1 to greater than 10 , with the variable domains of gp120 displaying the greatest diversity. $\mathbf{b}$ The Jensen-Shannon divergence between R5 and X4 gp120 sequence populations was computed for each amino acid position and plotted with a diamond. Statistically divergent positions $(\mathrm{P}<0.01)$ were plotted in red. A Monte Carlo permutation test was performed to iteratively group gp 120 sequences into random groups and calculate a distribution of expected Jensen-Shannon divergence values. The full range of this distribution was plotted in light blue with the interquartile range plotted in dark blue. The full range of divergence for randomly generated groups is in close agreement with the combined diversity of the R5 and X4 populations

of Jensen-Shannon divergence between the R5 and X4 populations identified 92 statistically significant positions throughout gp120 (Fig. 3b). A hypergeometric statistical test was used to determine if any domains of gp120 were enriched in statistically divergent positions, as opposed to the null hypothesis of equal distribution. Overall, the variable domains were enriched in divergent positions when compared to the null model, while the conserved domains were depleted, although the C3 and V5 domains were in slight opposition to this trend (Fig. 4). Specifically, the V3 loop was very highly enriched $\left[\log _{2}(\right.$ fold change $\left.)=1.89, \mathrm{P}=1.74 \times 10^{-11}\right]$ while the $\mathrm{C} 1$

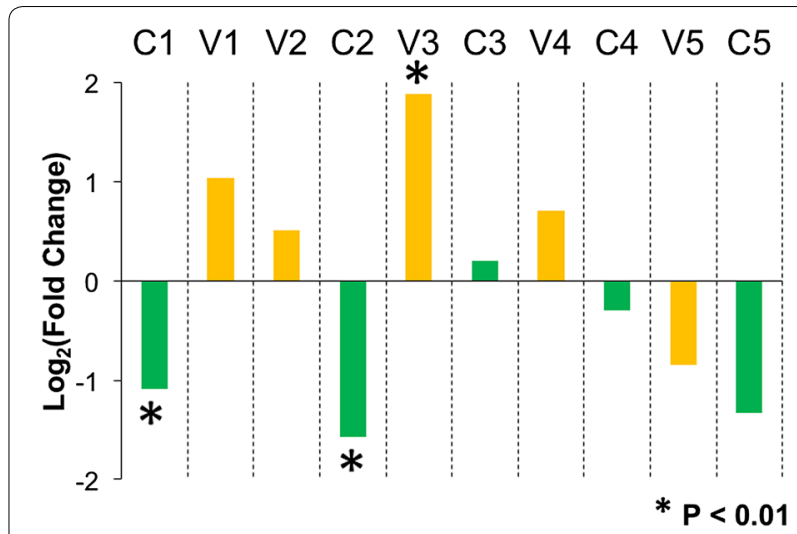

Fig. 4 V3 domain of gp120 is enriched with statistically divergent positions. The 10 conserved and variable domains of gp 120 were evaluated to determine if any regions were enriched in statistically divergent sites. A hypergeometric test was used to determine enrichment and depletion of statistically divergent positions, using the null hypothesis of equal distribution amongst domains. The V3 loop was identified as being highly enriched $\left(P=1.74 \times 10^{-11}\right)$, while the $\mathrm{C1}$ domain $\left(P=3.03 \times 10^{-4}\right)$ and $C 2$ domain $\left(P=1.28 \times 10^{-4}\right)$ were statistically depleted at $\mathrm{P}<0.01$ using a Benjamini-Hochberg multiple testing correction

domain $\left[\log _{2}(\right.$ fold change $\left.)=-1.09, \mathrm{P}=3.03 \times 10^{-4}\right]$ and C2 domain $\left[\log _{2}(\right.$ fold change $)=-1.57, \mathrm{P}=1.28 \times 10^{-4}$ ] were statistically depleted at $\mathrm{P}<0.01$ using a Benjamini-Hochberg multiple testing correction. With the understanding that gp120 and V3 behaved as expected following the application of diversity and divergence in this study, Tat and the LTR were investigated for similar signatures that may co-evolve with alterations in coreceptor utilization patterns exhibited by Env-V3.

\section{X4 Tat sequences demonstrate purifying selection in amino acid usage}

In general, we observed an overall trend for Tat to have higher amino acid diversity in the fifth and sixth domains of both groups (Fig. 5a). When we considered JensenShannon divergence, positions $7,23,57$, and 60 were found to be statistically divergent and $\mathrm{P}<0.01$ when accounting for multiple testing with the BenjaminiHochberg procedure (Fig. 5b). For all four divergent positions, the set of amino acids used in each population was similar, both with regard to the amino acids observed as well as the physiochemical properties of these amino acids (Fig. 6; Table 2). In all cases, the amino acid diversity of the X4 population was less than the diversity of the $\mathrm{R} 5$ population, demonstrating the qualitative trend that a subset of major variants become further enriched within the X4 population (Fig. 7). While there was no adequate statistical methodology to test the significance 


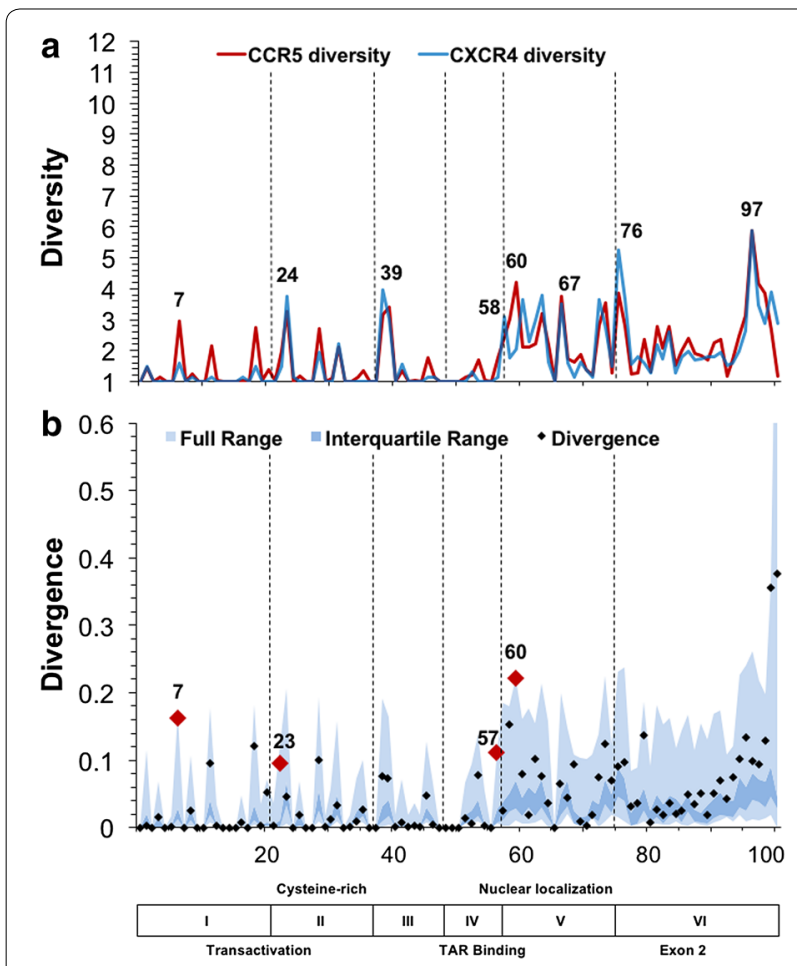

Fig. 5 Jensen-Shannon divergence identifies positions of differential amino acid usage between R5 and X4 HIV-1 Tat sequences. HIV-1 Tat sequences were sorted into $R 5(n=504)$ and $X 4(n=31)$ populations according to the predicted co-receptor usage of the co-linear V3 domain as determined by Web-PSSM score. a The diversity index at order $=1$ was calculated for each position for both R5 (red) and X4 (blue) Tat sequence populations. The diversity index between R5 and $X 4$ populations displayed high similarity at nearly all positions, with the second half of Tat displaying higher diversity values overall for both populations. b The Jensen-Shannon divergence between R5 and X4 Tat sequences was computed for each amino acid position and plotted with a diamond. Statistically divergent positions 7, 23, 57, and $60(P<0.01)$ were plotted in red and consensus changes, positions 40 and 67, were plotted in yellow. A Monte Carlo permutation test was performed to iteratively group Tat sequences into random groups and calculate a distribution of expected Jensen-Shannon divergence values. The full range of this distribution was plotted in light blue with the interquartile range plotted in dark blue of the difference in diversity at a single position, a Fisher's exact test was used to demonstrate the statistical enrichment of the consensus variants $\mathrm{R} 7(\mathrm{P}=0.00053), \mathrm{T} 23$ $(\mathrm{P}=0.0012)$, and $\mathrm{Q} 60(\mathrm{P}=0.0158)$ within the $\mathrm{X} 4$ group that is not a reflection of differences in R5 and X4 group sizes. In contrast, Tat variant R57 was not statistically enriched in the $\mathrm{X} 4$ population $(\mathrm{P}=0.17)$. Regardless, this observation lends support to a mechanism in which the HIV-1 Tat X4 genotype undergoes purifying selection in concert with the change in V3 co-receptor usage from R5 to X4, whereas $\mathrm{R} 5$ Tat may be able to persist within a less

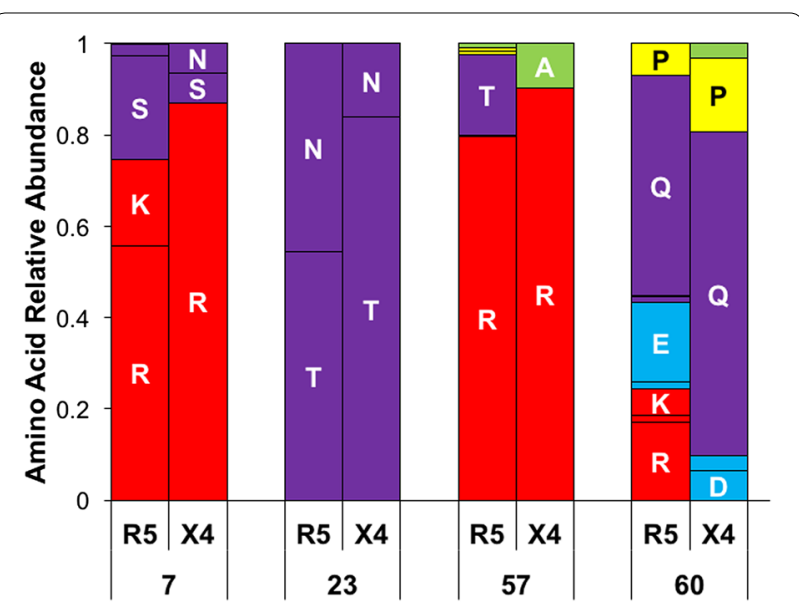

Fig. 6 Statistically divergent positions between X4 and R5 HIV-1 Tat interchange amino acids for those with similar physiochemical properties. The amino acid usage in four HIV-1 Tat amino acid positions (7, 23, 57, and 60) was plotted for both R5 and X4 groups as a stacked bar chart representing the total genetic variation within each population at the respective positions. Amino acids were color coded according to physiochemical property using the following scheme: positively charged (red), negatively charged (blue), polar uncharged (purple), hydrophobic (green), and unclassified (glycine, proline, and cysteine, yellow). The amino acid positions 7, 23, 57, and 60 were selected due to their statistically significant Jensen-Shannon divergence

constrained sequence space that allows it to effectively drive HIV-1 transcription in both T cells and MDMs and perhaps other cell lineages.

The four Tat amino acid positions of interest are located within domains responsible for transactivation, cysteine-rich, TAR binding, and nuclear localization, respectively (Fig. 5). In order to further evaluate the amino acid usage of these positions, the relative abundance of each variant was plotted (Fig. 6). In both R5 and $\mathrm{X} 4$ Tat, arginine is the most common variant at position 7 , although $\mathrm{R} 7$ is much more conserved within the X4 population. Previous studies have shown that an R7G substitution has only $93.9 \pm 6.5 \%$ of the transactivation activity of R7 [32]. While this effect size is small, it may contribute to differences in the transactivation activity between R5 and X4 HIV-1. Position 23 of Tat also is likely to play an important role in robust transactivation. In our data set, threonine was the most common variant observed at position 23, although asparagine was a frequent variant in both the R5 (46\%) and X4 (16\%) sequences. The T23N substitution has been shown to increase transactivation of the HIV-1 LTR, as well as binding to P-TEFb [33]. Accordingly, Tat N23 has been suggested to confer an advantage to HIV-1 by compensating for deleterious Tat mutations and supporting the 
Table 2 HIV-1 R5 and X4 Tat amino acid usage

\begin{tabular}{lrrrrr}
\hline Position & AA & R5 count & R5 \% & X4 count & X4 \% \\
\hline 7 & K & 95 & 0.19 & 0 & 0.00 \\
& N & 12 & 0.02 & 2 & 0.06 \\
& S & 115 & 0.23 & 2 & 0.06 \\
53 & R & 281 & 0.56 & 27 & 0.87 \\
& N & 230 & 0.46 & 5 & 0.16 \\
57 & T & 274 & 0.54 & 26 & 0.84 \\
& A & 5 & 0.01 & 3 & 0.10 \\
& R & 400 & 0.79 & 28 & 0.90 \\
60 & T & 89 & 0.18 & 0 & 0.00 \\
& E & 87 & 0.17 & 1 & 0.03 \\
& D & 7 & 0.01 & 2 & 0.06 \\
& K & 30 & 0.06 & 0 & 0.00 \\
& Q & 242 & 0.48 & 22 & 0.71 \\
& P & 35 & 0.07 & 5 & 0.16 \\
& R & 86 & 0.17 & 0 & 0.00 \\
\hline
\end{tabular}

The raw counts and relative abundance value of amino acids present in statistically divergent Tat positions for both the $\mathrm{R} 5$ and $\mathrm{X} 4$ sequence groups (7, $23,57$ and 60$)$

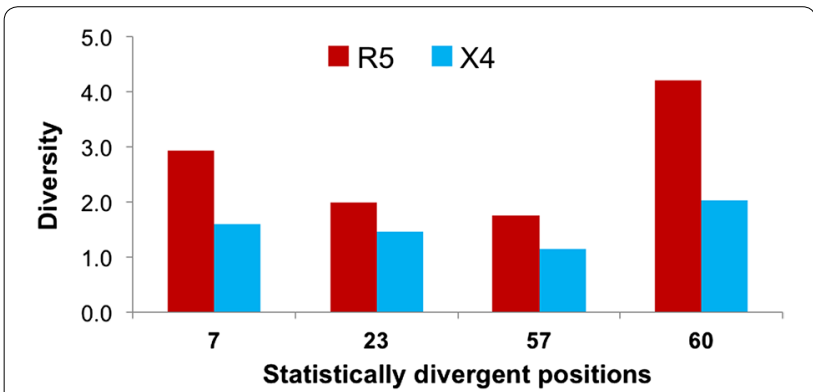

Fig. 7 Statistically divergent Tat positions demonstrate reduced diversity within X4-classified sequences. Within HIV-1 Tat, four amino acid positions were identified as having statistically significant Jensen-Shannon divergence: 7, 23, 57, and 60. In all four cases, it was noted that X4-classified variants exhibited a lower overall genetic diversity at an order of 1 , largely due to the enhanced presence of the most common variant in the X4 population. This pattern of diminished diversity within $X 4$ in comparison to R5 suggests that a purifying selective force may be present, affecting a subset of HIV-1 Tat variants

replication of less fit drug-resistant or immune-evasive quasispecies [33]. Furthermore, the enrichment of the T23N substitution in the R5 Tat population may relate to the ability of R5 HIV-1 to productively infect both T cells and monocytes and the selection of R5 HIV-1 at the time of transmission, as opposed to X4 HIV-1 which lacks a strong association with monocyte infection and is selected against at the time of transmission. The coselection and possible synergistic effect of these Tat variants remains an area of future investigation.
Predicted transcription factor binding sites have statistically different binding affinity scores between $\mathrm{X} 4$ and R5 LTR sequences

Genetic diversity and Jensen-Shannon divergence analyses were performed on LTR nucleotide sequences. 518 nucleotide positions spanning the HIV-1 U3, R, and U5 regions were evaluated. High levels of nucleotide diversity were present throughout the entire LTR and did not display a general pattern beyond being low, i.e. more highly conserved, in the approximately 50 nucleotides immediately downstream of the transcription start site that correspond to the TAR region of the LTR. This observation translated to large numbers of statistically significant Jensen-Shannon divergence scores $(n=48)$ between the two populations (Fig. 8). A number of these statistically divergent positions were identified at nucleotide positions within the core enhancer domain, the region of the LTR spanning approximately 200 nucleotides upstream of the transcription start site, while a high number of divergent positions were also identified in the less well characterized modulatory domain further upstream. Due to the high concentration of known transcription factor binding sites within the core enhancer domain, this region of the LTR was the focus of further analysis.

Binding of cellular transcription factors to the LTR has been shown to be one of the most critical parts of the viral life cycle with respect to acute infection but also in controlling the initial phases of genomic activation from latency. Throughout the HIV-1 LTR, eight well-known transcription factor binding sites were evaluated to determine if differential nucleotide usage exists between R5 and X4 populations: C/EBP-II (HXB2 positions 281-289), ATF-CREB (330-337), C/EBP-I (338-349), NF-кB-II (350-359), NF-кB-I (363-373), Sp-III (377386), Sp-II (388-398), and Sp-I (399-408). Additionally, the TAR stem-loop region (HXB2 positions 454-518) was similarly investigated for R5- and X4-associated differences.

The difference in nucleotide usage within known transcription factor binding sites was visualized using two-sequence logos (Fig. 9). This visualization creates a sequence logo for each transcription factor binding site that indicates nucleotides that are enriched within either the $\mathrm{R} 5$ or $\mathrm{X} 4$ populations, and scaled according to the maximum difference in relative abundance, such that nucleotides more frequently found in the R5 or X4 population are displayed on the bottom or top partition of the two-sequence logo, respectively, while completely conserved nucleotides are displayed in the middle. This analysis demonstrated that the greatest relative abundance differences in nucleotide usage occurred within sites C/EBP-I (54.7 \%), C/EBP-II (34.8\%), and Sp-III 


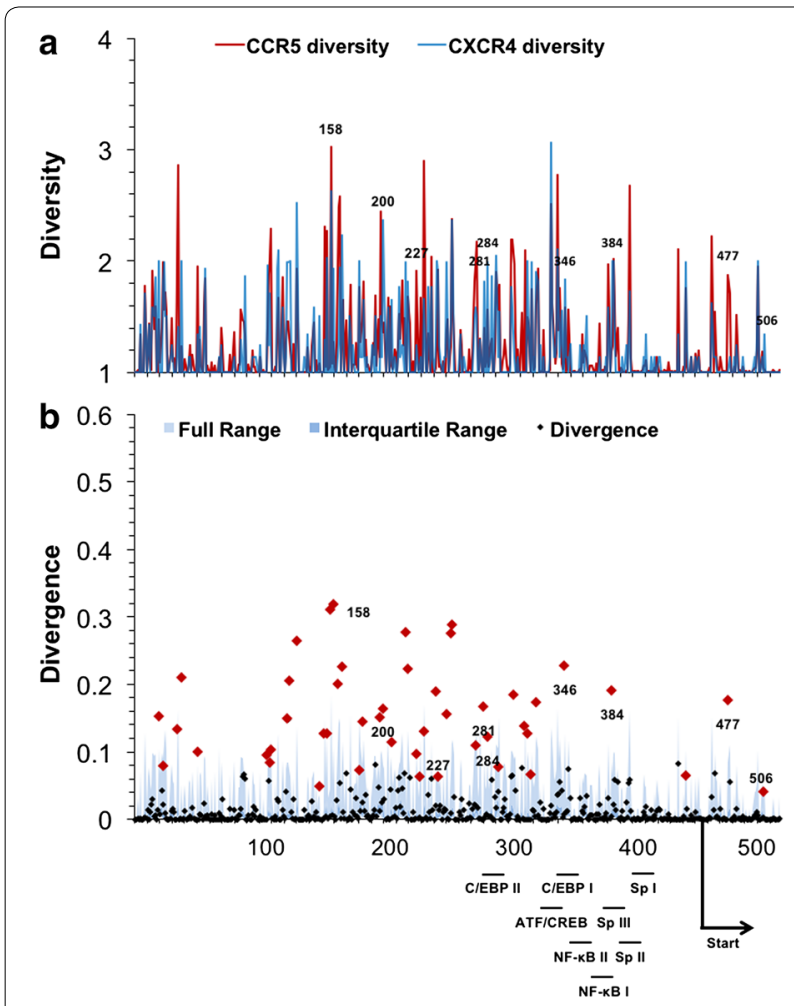

Fig. 8 HIV-1 LTR demonstrates high divergence both upstream and downstream of the transcription start site. HIV-1 long terminal repeat (LTR) sequences were sorted into R5 $(n=615)$ and X4 ( $=35)$ populations according to the predicted co-receptor usage of the co-linear V3 region. a The diversity index at order $=1$ was calculated for each position for both R5 (red) and X4 (blue) LTR sequence populations, numbered according to the HXB2 reference sequence. b Following the same approach applied for amino acid analysis, Jensen-Shannon divergence between R5 and X4 LTR sequences was computed for each nucleotide position and plotted. Statistically divergent positions were plotted in red and identified throughout the LTR, both upstream and downstream of the transcriptional start site and within transcription factor binding sites. A Monte Carlo permutation simulation was performed to randomly group LTR sequences and calculate a distribution of expected Jensen-Shannon divergence values, with the full range (light blue) and interquartile range (dark blue) of the distribution plotted across each position of the LTR

(43.2\%), as well as in the TAR stem loop (31.1\%). In contrast, NF-кB-I (11.7 \%), NF-кB-II (8.6 \%), Sp-I (13.8 \%), Sp-II (23.8\%), and ATF-CREB (11.8\%) showed rather modest differences between $\mathrm{X} 4$ and $\mathrm{R} 5$ in terms of nucleotide usage. Statistically divergent positions were identified within several of these transcription factor binding sites (Fig. 8), specifically sites C/EBP-I (position 346), C/ EBP-II (positions 281 and 284), and Sp-III (position 384), as well as position 477 of the TAR stem loop, in agreement with the maximal differences observed in the twosequence logos. Specifically, when comparing R5 to X4 sequences, the aforementioned positions demonstrated a

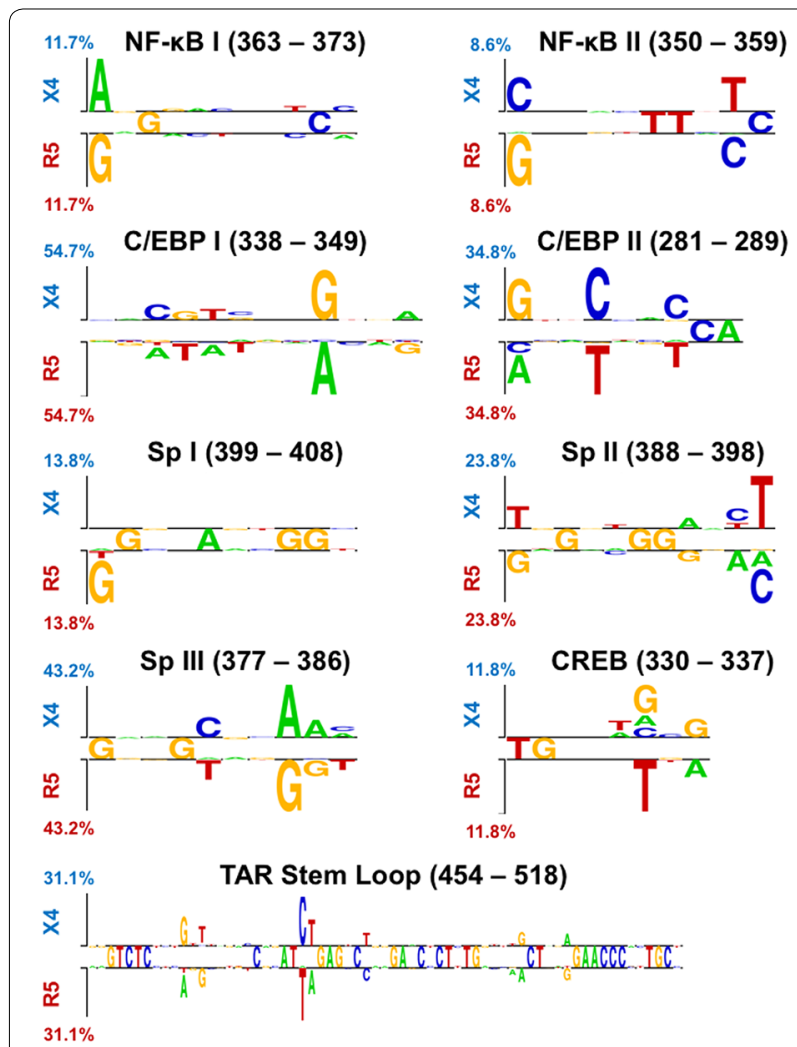

Fig. 9 R5 and X4 LTR sequences demonstrate signature enriched nucleotide variants in transcription factor binding. HIV-1 transcription factors that have been confirmed in vitro, C/EBP-II (HXB2 positions 281-289), ATF-CREB (330-337), C/EBP-I (338-349), NF-KB-II (350359), NF-KB-I (363-373), Sp-III (377-386), Sp-II (388-398), and Sp-I (399-408), as well as the TAR stem loop (454-518), were evaluated to detect enrichment and depletion of nucleotide variants in R5 and $X 4$ sets of aligned LTR sequences using two sample logos. Enriched nucleotides were plotted proportional to the difference between the populations, with the sum of the most differential position plotted on the vertical axis

propensity for an A-to-G (HXB2 position 346) mutation within C/EBP-I, an A/C-to-G (HXB2 position 281) and a T-to-C (HXB2 position 284) mutation within C/EBP-II, and a G-to-A (HXB2 position 384) mutation within SpIII. Finally, a large T-to-C (HXB2 position 477) mutation was observed within the bulge region of the TAR stem loop. The bulge region plays a crucial role in Tat recruitment and binding to the transcription complex, raising the possibility that X4 HIV-1 may contain a large subpopulation of genomes that have altered Tat recruitment and binding relative to R5 HIV-1 [34].

Although R5- and X4-specific nucleotide positions were identified throughout the LTR, it was not clear if those changes would result in meaningful differences between $\mathrm{R} 5$ and $\mathrm{X} 4$ in terms of transcription factor binding affinity. In order to quantitatively evaluate the 
difference between R5 and X4 LTR sequence groups, analysis was performed using the JASPAR database, a collection of transcription factor DNA-binding preferences modeled as matrices [35, 36]. Each LTR sequence was scanned for transcription factor binding sites by scoring against position weight matrices (PWMs), which were converted from JASPAR position frequency matrices (PFMs) downloaded from the JASPAR vertebrate database for C/EBP, SP1, NF-kB, and CREB. Each score was then compared to the maximum possible score of the corresponding PWM in order to determine a percentile score. Only binding sites with a mean percentile score $>0.30$ in either the R5- or X4-classified LTR sequences were considered for statistical analysis (Table 3). The distribution of R5- and X4-binding scores was statistically compared using a Kolmogorov-Smirnov (KS) test to calculate a $P$ value.

Using the JASPAR matrices, we were able to correctly identify the locations of each of the eight known transcription factor binding sites within the LTR when using a percentile scoring threshold of 0.3. At this threshold, the JASPAR matrices for C/EBP, Sp1, and CREB also identified several other potential novel binding locations (Table 3). Statistical analysis identified differential binding scores between the $\mathrm{R} 5$ and $\mathrm{X} 4$ populations at sites C/EBP-I, C/EBP-II, and Sp-III, but not at known transcription factor binding sites Sp-I or Sp-II (Table 3). This result is in agreement with the positions identified using Jensen-Shannon divergence. Additionally, novel putative Sp1 and C/EBP binding sites with differential R5/ X4 JAPSAR scores were noted at positions 223 and 150, respectively.

Interestingly, the relative magnitude of $\mathrm{R} 5$ and $\mathrm{X} 4$ mean binding scores of C/EBP-I and -II were opposite one another, with X4 LTRs having a greater mean binding score than R5 LTRs for C/EBP-I, whereas R5 LTRs have a greater mean binding score for C/EBP II. The novel putative C/EBP site followed the trend of C/EBP site I, and had a greater mean score among X4 LTRs. This relationship may be a compensatory effect by which the diminished binding affinity of C/EBP II, as the virus mutates from R5 to X4, leads to greater binding to $\mathrm{C} /$ EBP I and perhaps other putative C/EBP sites. This overall trend is also reflected among Sp binding sites. As the binding affinity of $\mathrm{Sp}$-III diminishes in $\mathrm{X} 4$ virus when compared to R5, a putative novel Sp site at position 223 gains enhanced binding affinity as indicated by JASPAR scoring. Generally, Sp sites have been shown to be more important for LTR-driven transcription in T lymphocytes than cells of the monocyte-macrophage lineage [37]. Furthermore, transcription factor binding at Sp-III varies with respect to the level of differentiation of monocytes [38]. Overall, we find that LTR-driven transcription is modulated by proteins of the $\mathrm{Sp}$ family in a manner that is specific to cell phenotype. As an important contrast, CREB and NF- $\mathrm{kB}$ binding sites did not demonstrate a difference in overall binding affinity between the R5 and X4 groups, although the analysis identified all known binding sites in both R5 and X4 populations. This is likely due to the fact that these sites have been shown to be essential for both T-lymphocyte and monocyte-macrophage replication [39-42].

\section{Conclusion}

The V3 domain of the HIV-1 env gene evolves throughout the course of infection, often resulting in a switch from an R5 to an X4 swarm. However, the characterization of R5 and X4 HIV-1 has not been defined beyond the envelope, specifically with respect to the transcriptional regulation of HIV-1. Our findings indicate that when comparing X4 HIV-1 to R5 HIV-1 sequences, Tat amino acids variants are more strictly selected at several key positions and specific LTR nucleotide variants are preferentially present in X4 HIV-1 sequences when compared to R5 HIV-1. One particular caveat of our analysis, and any that involves historical sequence review, is our choice of the functional annotation algorithm used in these studies. While the specific results may differ to some extent with the choice of computational tools used in a given study, the overall finding that there is co-evolution between gp120 and other regions of the HIV-1 genome remains consistent. These results are significant because they indicate that the transcriptional phenotype of HIV-1 may diverge with respect to co-receptor utilization. Importantly, the HIV-1 amino acid positions identified in Tat as different between X4 and R5 play roles in supporting robust transactivation, while the LTR nucleotide mutations associated with X4 and R5 strains are found within known and putative transcription factor binding sites and may affect their occupancy and contributions to the regulation of HIV-1 gene expression. We regard the genetic variation between X4 and R5 HIV-1 Tat and LTR sequences to be indicative of selection caused by the differential intracellular environments of cells preferentially infected by X4 versus R5 HIV-1 quasispecies. Consequently, the evolution of HIV-1 from an R5 to an $\mathrm{X} 4$ swarm likely requires adaptation at the level of transcriptional control in addition to co-receptor binding and entry.

\section{Methods}

\section{HIV-1 sequence collection}

HIV-1 sequences containing the Env-V3 region in addition to a co-linear Tat or LTR were collected and annotated from the Los Alamos National Laboratory (LANL) HIV Sequence Database as of October 2014, while 
Table 3 Predicted transcription factor binding sites in the HIV-1 LTR

\begin{tabular}{|c|c|c|c|c|c|c|c|}
\hline $\begin{array}{l}\text { Transcription } \\
\text { factor }\end{array}$ & $\begin{array}{l}\text { JASPAR } \\
\text { matrix }\end{array}$ & $\begin{array}{l}\text { In vitro } \\
\text { confirmed }\end{array}$ & $\begin{array}{l}\text { HXB2 } \\
\text { index }\end{array}$ & $\begin{array}{l}\text { Strand } \\
\text { direction }\end{array}$ & $\begin{array}{l}\text { X4 mean } \\
\text { score }\end{array}$ & $\begin{array}{l}\text { R5 mean } \\
\text { score }\end{array}$ & $P$ value \\
\hline \multirow[t]{12}{*}{ C/EBP } & MA0102.1-CEBPA & & 76 & Reverse & 4.55 & 4.06 & 0.3860 \\
\hline & MA0102.2-CEBPA & & 79 & Reverse & 4.94 & 4.41 & 0.3860 \\
\hline & MA0102.2-CEBPA & & 81 & Forward & 4.24 & 3.12 & 0.2304 \\
\hline & MA0102.1-CEBPA & & 150 & Reverse & 5.16 & 3.39 & $<0.0001$ \\
\hline & MA0102.2-CEBPA & & 153 & Reverse & 6.35 & 4.58 & $<0.0001$ \\
\hline & MA0102.2-CEBPA & & 154 & Forward & 4.53 & 1.83 & $<0.0001$ \\
\hline & MA0102.1-CEBPA & & 197 & Reverse & 5.27 & 4.63 & 0.0092 \\
\hline & MA0102.2-CEBPA & & 200 & Reverse & 4.33 & 4.34 & 0.0063 \\
\hline & MA0102.1-CEBPA & $C / E B P \|$ & 278 & Reverse & 5.84 & 6.29 & 0.0005 \\
\hline & MA0102.2-CEBPA & $C / E B P \|$ & 281 & Reverse & 5.26 & 5.70 & 0.0010 \\
\hline & MA0102.3-CEBPA & C/EBP II & 281 & Forward & 7.65 & 9.67 & 0.0008 \\
\hline & MA0102.2-CEBPA & $C / E B P I$ & 342 & Forward & 5.26 & 4.65 & $<0.0001$ \\
\hline \multirow[t]{3}{*}{ CREB } & MA0018.2-CREB1 & & 173 & Forward & 4.74 & 3.93 & 0.1020 \\
\hline & MA0018.2-CREB1 & ATF/CREB & 330 & Forward & 5.25 & 5.63 & 0.4547 \\
\hline & MA0018.2-CREB1 & & 410 & Reverse & 4.43 & 4.68 & 0.8684 \\
\hline \multirow[t]{16}{*}{ NF-kB } & MA0105.1-NFKB1 & NF-kB II & 350 & Forward & 13.66 & 14.52 & 0.9605 \\
\hline & MA0105.1-NFKB1 & NF-kB II & 350 & Reverse & 7.43 & 8.30 & 0.9605 \\
\hline & MA0105.2-NFKB1 & NF-kB II & 350 & Forward & 6.67 & 7.41 & 0.9605 \\
\hline & MA0105.3-NFKB1 & NF-kB II & 350 & Forward & 12.37 & 13.41 & 0.9722 \\
\hline & MA0105.1-NFKB1 & NF-kB II & 351 & Reverse & 4.85 & 5.31 & 0.9813 \\
\hline & MA0105.1-NFKB1 & NF-kB I & 363 & Forward & 6.18 & 6.77 & 0.8062 \\
\hline & MA0105.2-NFKB1 & NF-kB I & 363 & Forward & 8.20 & 8.62 & 0.8062 \\
\hline & MA0105.2-NFKB1 & NF-kB I & 363 & Reverse & 7.60 & 7.88 & 0.8345 \\
\hline & MA0105.1-NFKB1 & NF-kB I & 364 & Forward & 14.55 & 14.35 & 1.0000 \\
\hline & MA0105.1-NFKB1 & NF-kB I & 364 & Reverse & 8.33 & 8.26 & 1.0000 \\
\hline & MA0105.2-NFKB1 & NF-kB I & 364 & Forward & 7.44 & 7.29 & 1.0000 \\
\hline & MA0105.3-NFKB1 & NF-kB I & 364 & Forward & 15.66 & 15.15 & 0.8907 \\
\hline & MA0105.1-NFKB1 & NF-kB I & 365 & Reverse & 5.34 & 5.15 & 1.0000 \\
\hline & MA0105.2-NFKB1 & NF-kB & 504 & Forward & 6.09 & 6.46 & 0.9850 \\
\hline & MA0105.2-NFKB1 & NF-kB & 504 & Reverse & 8.56 & 8.83 & 0.9881 \\
\hline & MA0105.1-NFKB1 & NF-kB & 505 & Reverse & 6.01 & 6.30 & 0.9850 \\
\hline \multirow[t]{18}{*}{ Sp } & MA0079.2-SP1 & & 98 & Reverse & 4.68 & 5.68 & 0.0093 \\
\hline & MA0079.1-SP1 & & 99 & Forward & 5.01 & 5.35 & 0.0093 \\
\hline & MA0079.1-SP1 & & 223 & Forward & 4.35 & 3.31 & $<0.0001$ \\
\hline & MA0079.1-SP1 & & 224 & Forward & 4.57 & 2.05 & $<0.0001$ \\
\hline & MA0079.1-SP1 & & 266 & Reverse & 4.36 & 3.37 & 0.1034 \\
\hline & MA0079.2-SP1 & Sp-III & 373 & Reverse & 5.30 & 5.86 & 0.5696 \\
\hline & MA0079.1-SP1 & Sp--III & 374 & Forward & 4.12 & 4.82 & 0.0755 \\
\hline & MA0079.2-SP1 & Sp-III & 376 & Reverse & 5.22 & 6.46 & $<0.0001$ \\
\hline & MA0079.1-SP1 & $S p-I I I$ & 377 & Forward & 3.86 & 5.56 & $<0.0001$ \\
\hline & MA0079.2-SP1 & $S p-I I I$ & 382 & Reverse & 4.71 & 5.81 & $<0.0001$ \\
\hline & MA0079.2-SP1 & Sp-II & 387 & Reverse & 6.72 & 7.11 & 0.7894 \\
\hline & MA0079.3-SP1 & Sp-II & 387 & Reverse & 10.11 & 10.46 & 0.6982 \\
\hline & MA0079.1-SP1 & Sp-II & 388 & Forward & 5.78 & 6.14 & 0.2648 \\
\hline & MA0079.2-SP1 & Sp-II & 392 & Reverse & 6.62 & 7.07 & 0.1179 \\
\hline & MA0079.2-SP1 & Sp-II & 393 & Reverse & 5.51 & 6.81 & 0.0168 \\
\hline & MA0079.1-SP1 & Sp-1 & 398 & Forward & 4.35 & 4.35 & 0.6176 \\
\hline & MA0079.2-SP1 & Sp-1 & 398 & Reverse & 6.69 & 7.21 & 0.5383 \\
\hline & MA0079.1-SP1 & Sp-I & 399 & Forward & 4.30 & 4.47 & 0.5539 \\
\hline
\end{tabular}


Table 3 continued

\begin{tabular}{lllllrr}
\hline $\begin{array}{l}\text { Transcription } \\
\text { factor }\end{array}$ & $\begin{array}{l}\text { JASPAR } \\
\text { matrix }\end{array}$ & $\begin{array}{l}\text { In vitro } \\
\text { confirmed }\end{array}$ & $\begin{array}{l}\text { HXB2 } \\
\text { index }\end{array}$ & $\begin{array}{l}\text { Strand } \\
\text { direction }\end{array}$ & $\begin{array}{l}\text { X4 mean } \\
\text { score }\end{array}$ & $\begin{array}{l}\text { R5 mean } \\
\text { score }\end{array}$ \\
\hline & MA0079.2-SP1 & Sp-I & 400 & Reverse & 5.78 \\
& MA0079.1-SP1 & & 479 & Forward & 5.21 & 5.0000
\end{tabular}

LTR sequences classified as either R5 or X4 based on their co-linear Env-V3 sequence were scanned for potential transcription factor binding sites. All binding sites with a percentile score $>0.3$ in either of the two groups were included in this analysis. The overall score distribution of R5 and X4 binding sites was compared using a KS-test, and multiple testing was accounted for using the Benjamini-Hochberg procedure. Statistically significant sites $(\mathrm{P}<0.01)$ are highlighted in italics

additional sequences from the Drexel Medicine CNS Research and AIDS Eradication Study (CARES) Cohort were added to supplement the total number of sequences publicly available. The Drexel Medicine CARES Cohort is a subtype B patient cohort from Philadelphia, Pennsylvania and has been previously described [43-46]. The sequences from the Drexel Medicine CARES Cohort have been submitted to Genbank under BioProject ID: PRJNA319822. To reduce the effect of regional and subtype differences, the LANL database query was limited to include only subtype B sequences isolated from North America. The query was further limited to a single sequence per patient using the LANL query tool which specifically excludes laboratory strain sequences or those used for functional studies. Table 1 shows the breakdown of sequences for each region.

\section{Co-receptor usage classification}

The in silico co-receptor usage prediction tool WebPSSM was used to classify all sequences as CCR5- or CXCR4-utilizing based on the score of the co-linear Env-V3 amino acid sequence [17]. Numerous exclusion methods were utilized to reduce noise introduced by Web-PSSM predictions as discussed previously [47]. Sequences were excluded from the study if the V3 region was not 35 amino acid residues in length, if the V3 percentile determined by Web-PSSM was greater than 0.95 (indicating that a given sequence may not be a V3 sequence), or if the V3 PSSM score was in the 'indeterminate range' (using scoring cutoffs of $>-2.88$ and $<-6.96$ for $\mathrm{X} 4$ and R5 Env-V3 sequences, respectively), which was defined as a scoring range consisting of sequences with $\mathrm{R} 5$ and/or $\mathrm{X} 4$ properties including sequences that are dual tropic (X4/R5). Using these cutoffs, this predictor has an $84 \%$ sensitivity and $96 \%$ specificity indicating its ability to detect X4 binding sequences and non-binding sequences, respectively [17]. This filtering method allowed the genetic analysis to focus on sequences with the highest confidence classification in the PSSM-derived distribution, definitively signifying CCR5- or CXCR4utilizing Env-V3 sequences. Following classification as R5 or X4, the co-linear gp120, Tat, and LTR sequences were aligned to the HXB2 reference sequence (K03455) using Multiple Sequence Comparison by Log-Expectation
(MUSCLE), (version 5.05) [48] utilizing default parameters; insertions relative to the reference were removed to simplify the analysis. This pipeline resulted in R5- and $\mathrm{X} 4$-associated and multiple sequence alignments for each gp120, Tat, and LTR sequence (Table 1).

\section{Genetic diversity and rarefaction}

The diversity of each amino acid or nucleotide position of the respective multiple sequence alignments was calculated using a window length, $w$, of 1 and an order of 1 [equivalent to $\exp$ (Shannon entropy with base e)] according to Eq. 1 [49].

First-order genetic diversity

$$
\mathrm{D}_{\mathrm{w}, \mathrm{p}}=\exp \left(-\sum_{\mathrm{i}=1}^{\mathrm{R}_{\mathrm{w}, \mathrm{p}}} \mathrm{p}_{\mathrm{i}_{\mathrm{w}, \mathrm{p}}} \ln \left[\mathrm{p}_{\mathrm{i}_{\mathrm{w}, \mathrm{p}}}\right]\right)
$$

Diversity, $D$, weighs the abundance of all variants, $p$, at a given position, $i$, in the protein. A window length, $w$, is applied, with $w=1$ used in order to independently assess the diversity of each position within a multiple sequence alignment. At an order, or Hill number, of $\mathrm{q}=1, D$ does not exist; however, the limit as $q$ approaches 1 can be computed as shown here.

Diversity at order $=1$ calculates the effective number of species (amino acids or nucleotides) in a population while giving greater weight to neither rare nor abundant species. The maximum possible diversity is 20 for amino acid sequences (gp120 and Tat) and 4 for nucleotide sequences (LTR), with gaps regarded as missing data. In general, positions of high structural or functional importance are evolutionarily constrained in their use of amino acids or nucleotides and therefore demonstrate low diversity, while positions more permissive to variation in amino acid or nucleotide usage displayed higher genetic diversity [50]. Rarefaction curves for each position were generated in order to ensure that sufficient sample sizes existed for each comparison being made.

\section{Jensen-Shannon divergence}

Jensen-Shannon divergence is a measure of the similarity between two probability distributions that can be applied to profile-to-profile multiple sequence alignment 
comparisons, with the divergence score bound by 0 (similar) and 1 (dissimilar) [51, 52]. Multiple sequence alignments (MSA) generated from R5- and X4-classified sequence populations were used to generate position frequency matrices (PFMs). Each PFM contains the relative abundance of each residue (amino acid or nucleotide) for each position $(\mathrm{N})$ of the multiple sequence alignment, resulting in $20 \times \mathrm{N}$ or $4 \times \mathrm{N}$ matrices for amino acid or nucleotide sequences, respectively. Residues that are not present in any of the sequences at a particular position of the MSA were represented with a pseudo-count of $1 \times 10^{-7}$, equivalent to a relative abundance of 1 instance per ten million sequences, which ranges from approximately $1 \times 10^{4}$-fold to $1 \times 10^{6}$-fold lower abundance than being present in a single sequence. PFMs derived from R5- and X4-classified sequences were used to calculate the Jensen-Shannon divergence between populations according to Eq. 2.

Jensen-Shannon divergence

$$
\mathrm{D}_{\text {IS }}=\frac{1}{2}\left[\sum_{\mathrm{a}=1}^{20} \mathrm{Q}_{\mathrm{a}}^{1} \log _{2} \frac{\mathrm{Q}_{\mathrm{a}}^{1}}{\mathrm{Q}_{\mathrm{a}}^{0}}+\sum_{\mathrm{a}=1}^{20} \mathrm{Q}_{\mathrm{a}}^{2} \log _{2} \frac{\mathrm{Q}_{\mathrm{a}}^{2}}{\mathrm{Q}_{\mathrm{a}}^{0}}\right]
$$

where

$$
\mathrm{Q}_{\mathrm{a}}^{0}=\frac{1}{2}\left(\mathrm{Q}_{\mathrm{a}}^{1}+\mathrm{Q}_{\mathrm{a}}^{2}\right)
$$

Jensen-Shannon divergence, $D_{J S}$, is determined according to the abundance of each amino acid variant, $Q_{a}$, in populations 1 and 2, using an information theory-based calculation. The value $Q^{0}$ is calculated for each amino acid variant, and a pseudo-count is utilized for amino acid variants absent in both populations.

Statistically significant positions were identified by applying a Monte Carlo permutation test, which randomly re-grouped the total pool of sequences into groups of size $\mathrm{M}$ and $\mathrm{N}$ iteratively ( $\mathrm{n}=1000)$, where $\mathrm{M}$ and $\mathrm{N}$ are equivalent to the number of sequences in the $\mathrm{X} 4$ and R5 groups, and generated a probability density function (PDF) of the Jensen-Shannon divergence values of the randomized model using a Gaussian kernel density estimator implemented in SciPy. Numerical integration was used to determine the probability of finding a random value greater than or equal to the true Jensen-Shannon divergence.

\section{Statistical analysis}

Statistical analysis was performed in custom IPython Notebooks using the SciPy Python library (version 0.14.0). Spearman's rank correlation coefficient was used to evaluate the relationship between R5 and X4 diversity (Fig. 1), as well as the relationship of Jensen-Shannon divergence to mean genetic diversity and the absolute difference in genetic diversity (Fig. 2), respectively. gp120 domain enrichment analysis (Fig. 4) of statistically divergent positions utilized a hypergeometric test, with a null hypothesis of equal distribution of divergent positions. Enrichment of consensus amino acids within statistically divergent Tat positions was performed using a Fisher's exact test (Fig. 6).

\section{Two sequence logos}

Experimentally validated transcription factor bindings sites C/EBP-II (HXB2 positions 281-289), ATF-CREB (330-337), C/EBP-I (338-349), NF-kB-II (350-359), NF-кB-I (363-373), Sp-III (377-386), Sp-II (388-398), and Sp-I (399-408), as well as the RNA stem loop (454518), were evaluated using Two Sample Logo [53]. Two Sample Logo is a web-based application that calculates and visualizes the differences between two sets of aligned sequences. Each nucleotide was represented with a different color, and the height of the one-letter nucleotide code was scaled according to the magnitude of the difference in abundance of the nucleotide at a given position, with the largest difference in each comparison represented by the maximum height in the logo.

\section{Identification of putative transcription binding sites}

Position frequency matrices (PFMs) were downloaded from the JASPAR redundant vertebrate database for $\mathrm{C} /$ EBP, Sp, NFkB, and CREB. Each PFM was converted into a position weight matrix (PWM) as previously described [52]. Each LTR sequence was scanned along its entirety to score every potential binding site using each of the PWMs. Each score was then compared to the maximum possible score for the PWM being used in order to determine a percentile score. Only binding sites with a mean percentile score $>0.30$ in either the R5- or X4-classified LTR sequences were considered for statistical analysis (Table 3). Binding affinities as defined by PWM score show a non-Gaussian distribution (data not shown). As such, the Kolmogorov-Smirnov (KS) test was used to compare affinities between different groups. The PWM was applied to each LTR and then the R5 and X4 distributions were compared. The $P$ values were adjusted using the Benjamini-Hochberg procedure.

\section{Authors' contributions \\ GA, BA, WD, MN, UH and BW conceived and designed the study. GA, WD, BA, $G S$, and $U H$ designed and performed the bioinformatic and statistical analyses. BW, MN, VP, JJ, KK, JW, WZ, BA, GA and SP managed the Drexel Medicine CARES Cohort and/or obtained sequences from patient PBMC samples. GA, UH, MN, FK, and BW prepared and designed the figures and drafted the manuscript. All authors read and approved the final manuscript.}

\section{Author details}

${ }^{1}$ Department of Microbiology and Immunology, Drexel University College of Medicine, Philadelphia, PA, USA. ${ }^{2}$ Center for Molecular Virology and Translational Neuroscience, Institute for Molecular Medicine and Infectious 
Disease, Drexel University College of Medicine, Philadelphia, PA, USA. ${ }^{3}$ School of Biomedical Engineering, Science, and Health Systems, Drexel University, Philadelphia, PA, USA. ${ }^{4}$ Division of Infectious Diseases and HIV Medicine, Department of Medicine, Drexel University College of Medicine, Philadelphia, PA, USA. ${ }^{5}$ Center for Clinical and Translational Medicine, Institute for Molecular Medicine and Infectious Disease, Drexel University College of Medicine, Philadelphia, PA, USA. ${ }^{6}$ Sidney Kimmel Cancer Center, Thomas Jefferson University, Philadelphia, PA, USA.

\section{Acknowledgements}

We would like to thank all patients who are part of the Drexel Medicine CARES Cohort. We would also like to thank the clinical staff within the Division of Infectious Diseases and HIV Medicine and Center for Clinical and Translational Medicine in the Institute for Molecular Medicine and Infectious Disease at the Drexel University College of Medicine who are involved in recruitment, enrollment, obtaining consent, obtaining clinical histories, venipuncture, and delivery of peripheral blood to the research laboratories in the Center for Molecular Virology and Translational Neuroscience in the Institute for Molecular Medicine and Infectious Disease. These studies were funded in part by the Public Health Service, National Institutes of Health, through grants from the National Institute of Neurological Disorders and Stroke (NS32092 and NS46263, Dr. Brian Wigdahl, Principal Investigator; NS089435, Dr. Michael R. Nonnemacher, Principal Investigator), the National Institute of Drug Abuse (DA19807, Dr. Brian Wigdahl, Principal Investigator), National Institute of Mental Health Comprehensive NeuroAIDS Center (CNAC) (P30 MH-092177, Kamel Khalili, PI; Brian Wigdahl, Pl of the Drexel subcontract; Michael Nonnemacher, $\mathrm{Pl}$, Developmental Grant), and under the Ruth L. Kirschstein National Research Service Award 5T32MH079785 (Jay Rappaport, Pl; Brian Wigdahl, Pl of the Drexel subcontract). The contents of the paper are solely the responsibility of the authors and do not necessarily represent the official views of the NIH. Drs. Michael Nonnemacher, Will Dampier, and Fred Krebs were also supported by faculty development funds provided by the Department of Microbiology and Immunology and the Institute for Molecular Medicine and Infectious Disease.

\section{Competing interests}

The authors declare that they have no competing interests.

\section{Ethics statement}

The Drexel University College of Medicine Institutional Review Board (IRB) has approved this work under protocol 1201000748. All patient samples were collected under the auspices of this protocol through written consent. Dr. Brian Wigdahl is PI of the IRB protocol.

Received: 1 December 2015 Accepted: 20 April 2016 Published online: 03 May 2016

\section{References}

1. Arrildt KT, Joseph SB, Swanstrom R. The HIV-1 env protein: a coat of many colors. Curr HIV/AIDS Rep. 2012;9:52-63.

2. Sirois S, Sing T, Chou KC. HIV-1 gp120 V3 loop for structure-based drug design. Curr Protein Pept Sci. 2005;6:413-22.

3. Javaherian K, Langlois AJ, McDanal C, Ross KL, Eckler LI, Jellis CL, Profy AT, Rusche JR, Bolognesi DP, Putney SD, et al. Principal neutralizing domain of the human immunodeficiency virus type 1 envelope protein. Proc Natl Acad Sci USA. 1989;86:6768-72.

4. Goudsmit J, Debouck C, Meloen RH, Smit L, Bakker M, Asher DM, Wolff AV, Gibbs CJ Jr, Gajdusek DC. Human immunodeficiency virus type 1 neutralization epitope with conserved architecture elicits early type-specific antibodies in experimentally infected chimpanzees. Proc Natl Acad Sci USA. 1988;85:4478-82.

5. Sharon M, Kessler N, Levy R, Zolla-Pazner S, Gorlach M, Anglister J. Alternative conformations of HIV-1 V3 loops mimic beta hairpins in chemokines, suggesting a mechanism for coreceptor selectivity. Structure. 2003;11:225-36

6. De Jong JJ, De Ronde A, Keulen W, Tersmette M, Goudsmit J. Minimal requirements for the human immunodeficiency virus type $1 \mathrm{~V} 3$ domain to support the syncytium-inducing phenotype: analysis by single amino acid substitution. J Virol. 1992;66:6777-80.
7. Cormier EG, Dragic T. The crown and stem of the $\mathrm{V} 3$ loop play distinct roles in human immunodeficiency virus type 1 envelope glycoprotein interactions with the CCR5 coreceptor. J Virol. 2002;76:8953-7.

8. Fouchier RA, Groenink M, Kootstra NA, Tersmette M, Huisman HG, Miedema F, Schuitemaker H. Phenotype-associated sequence variation in the third variable domain of the human immunodeficiency virus type 1 gp120 molecule. J Virol. 1992;66:3183-7.

9. Feng Y, Broder CC, Kennedy PE, Berger EA. HIV-1 entry cofactor: functional cDNA cloning of a seven-transmembrane, G protein-coupled receptor. Science. 1996;272:872-7.

10. Deng H, Liu R, Ellmeier W, Choe S, Unutmaz D, Burkhart M, Di Marzio P, Marmon S, Sutton RE, Hill CM, et al. Identification of a major co-receptor for primary isolates of HIV-1. Nature. 1996;381:661-6.

11. Swanstrom R, Coffin J. HIV-1 pathogenesis: the virus. Cold Spring Harb Perspect Med. 2012;2:a007443.

12. Gonzalez-Scarano F, Martin-Garcia J. The neuropathogenesis of AIDS. Nat Rev Immunol. 2005;5:69-81.

13. Ochsenbauer C, Edmonds TG, Ding H, Keele BF, Decker J, Salazar MG, Salazar-Gonzalez JF, Shattock R, Haynes BF, Shaw GM, et al. Generation of transmitted/founder HIV-1 infectious molecular clones and characterization of their replication capacity in CD4 T lymphocytes and monocytederived macrophages. J Virol. 2012;86:2715-28.

14. Keele BF, Giorgi EE, Salazar-Gonzalez JF, Decker JM, Pham KT, Salazar MG, Sun C, Grayson T, Wang S, Li H, et al. Identification and characterization of transmitted and early founder virus envelopes in primary HIV-1 infection. Proc Natl Acad Sci USA. 2008;105:7552-7.

15. Sierra S, Kaiser R, Thielen A, Lengauer T. Genotypic coreceptor analysis. Eur J Med Res. 2007;12:453-62.

16. Verhofstede C, Brudney D, Reynaerts J, Vaira D, Fransen K, De Bel A, Seguin-Devaux C, De Wit S, Vandekerckhove L, Geretti AM. Concordance between HIV-1 genotypic coreceptor tropism predictions based on plasma RNA and proviral DNA. HIV Med. 2011;12:544-52.

17. Jensen MA, Li FS, van't Wout AB, Nickle DC, Shriner D, He HX, McLaughlin S, Shankarappa R, Margolick JB, Mullins JI. Improved coreceptor usage prediction and genotypic monitoring of R5-to-X4 transition by motif analysis of human immunodeficiency virus type 1 env $\mathrm{V} 3$ loop sequences. J Virol. 2003;77:13376-88.

18. Aiamkitsumrit B, Dampier W, Antell G, Rivera N, Martin-Garcia J, Pirrone V, Nonnemacher MR, Wigdahl B. Bioinformatic analysis of HIV-1 entry and pathogenesis. Curr HIV Res. 2014;12:132-61.

19. Carrillo A, Ratner L. Cooperative effects of the human immunodeficiency virus type 1 envelope variable loops $\mathrm{V} 1$ and $\mathrm{V} 3$ in mediating infectivity for T cells. J Virol. 1996;70:1310-6.

20. Huang W, Toma J, Fransen S, Stawiski E, Reeves JD, Whitcomb JM, Parkin N, Petropoulos CJ. Coreceptor tropism can be influenced by amino acid substitutions in the gp41 transmembrane subunit of human immunodeficiency virus type 1 envelope protein. J Virol. 2008;82:5584-93.

21. Koito A, Stamatatos L, Cheng-Mayer C. Small amino acid sequence changes within the $\mathrm{V} 2$ domain can affect the function of a T-cell linetropic human immunodeficiency virus type 1 envelope gp120. Virology. 1995;206:878-84.

22. Labrosse B, Treboute C, Brelot A, Alizon M. Cooperation of the V1/N2 and V3 domains of human immunodeficiency virus type $1 \mathrm{gp} 120$ for interaction with the CXCR4 receptor. J Virol. 2001;75:5457-64.

23. Pastore C, Nedellec R, Ramos A, Pontow S, Ratner L, Mosier DE. Human immunodeficiency virus type 1 coreceptor switching: $V 1$ N2 gain-of-fitness mutations compensate for V3 loss-of-fitness mutations. J Virol. 2006;80:750-8

24. Suphaphiphat $P$, Essex M, Lee TH. Mutations in the $V 3$ stem versus the $V 3$ crown and $C 4$ region have different effects on the binding and fusion steps of human immunodeficiency virus type $1 \mathrm{gp} 120$ interaction with the CCR5 coreceptor. Virology. 2007;360:182-90.

25. Thielen A, Sichtig N, Kaiser R, Lam J, Harrigan PR, Lengauer T. Improved prediction of HIV-1 coreceptor usage with sequence information from the second hypervariable loop of gp120. J Infect Dis. 2010;202:1435-43.

26. Dimonte S, Mercurio F, Svicher V, D'Arrigo R, Perno CF, Ceccherini-Silberstein F. Selected amino acid mutations in HIV-1 B subtype gp41 are associated with specific gp120v(3) signatures in the regulation of co-receptor usage. Retrovirology. 2011;8:33.

27. Coetzer M, Nedellec R, Cilliers T, Meyers T, Morris L, Mosier DE. Extreme genetic divergence is required for coreceptor switching in HIV-1 subtype C. J Acquir Immune Defic Syndr. 2011;56:9-15. 
28. Kwong PD, Wyatt R, Robinson J, Sweet RW, Sodroski J, Hendrickson WA. Structure of an HIV gp120 envelope glycoprotein in complex with the CD4 receptor and a neutralizing human antibody. Nature. 1998;393:648-59.

29. Nonnemacher MR, Irish BP, Liu Y, Mauger D, Wigdahl B. Specific sequence configurations of HIV-1 LTR G/C box array result in altered recruitment of Sp isoforms and correlate with disease progression. J Neuroimmunol. 2004;157:39-47.

30. Roy S, Delling U, Chen CH, Rosen CA, Sonenberg N. A bulge structure in HIV-1 TAR RNA is required for Tat binding and Tat-mediated trans-activation. Genes Dev. 1990:4:1365-73.

31. Sturdevant CB, Joseph SB, Schnell G, Price RW, Swanstrom R, Spudich S. Compartmentalized replication of R5 T cell-tropic HIV-1 in the central nervous system early in the course of infection. PLoS Pathog. 2015;11:e1004720.

32. Yukl S, Pillai S, Li P, Chang K, Pasutti W, Ahlgren C, Havlir D, Strain M, Gunthard H, Richman D, et al. Latently-infected CD4 + T cells are enriched for HIV-1 Tat variants with impaired transactivation activity. Virology. 2009;387:98-108.

33. Reza SM, Shen LM, Mukhopadhyay R, Rosetti M, Pe'ery T, Mathews MB. A naturally occurring substitution in human immunodeficiency virus Tat increases expression of the viral genome. J Virol. 2003;77:8602-6.

34. Selby MJ, Bain ES, Luciw PA, Peterlin BM. Structure, sequence, and position of the stem-loop in tar determine transcriptional elongation by tat through the HIV-1 long terminal repeat. Genes Dev. 1989;3:547-58.

35. Sandelin A, Alkema W, Engstrom P, Wasserman WW, Lenhard B. JASPAR: an open-access database for eukaryotic transcription factor binding profiles. Nucleic Acids Res. 2004;32:D91-4.

36. Mathelier A, Zhao X, Zhang AW, Parcy F, Worsley-Hunt R, Arenillas DJ, Buchman S, Chen CY, Chou A, lenasescu H, et al. JASPAR 2014: an extensively expanded and updated open-access database of transcription factor binding profiles. Nucleic Acids Res. 2014;42:D142-7.

37. McAllister JJ, Phillips D, Millhouse S, Conner J, Hogan T, Ross HL, Wigdahl B. Analysis of the HIV-1 LTR NF-kappaB-proximal Sp site III: evidence for cell type-specific gene regulation and viral replication. Virology. 2000;274:262-77.

38. Millhouse S, Krebs FC, Yao J, McAllister JJ, Conner J, Ross H, Wigdahl B. Sp1 and related factors fail to interact with the NF-kappaB-proximal G/C box in the LTR of a replication competent, brain-derived strain of HIV-1 (YU-2). J Neurovirol. 1998;4:312-23.

39. Palmieri C, Trimboli F, Puca A, Fiume G, Scala G, Quinto I. Inhibition of HIV-1 replication in primary human monocytes by the IkappaBalphaS32/36A repressor of NF-kappaB. Retrovirology. 2004;1:45.

40. Rohr O, Sawaya BE, Lecestre D, Aunis D, Schaeffer E. Dopamine stimulates expression of the human immunodeficiency virus type 1 via NF-kappaB in cells of the immune system. Nucleic Acids Res. 1999;27:3291-9.
41. Rohr O, Aunis D, Schaeffer E. COUP-TF and Sp1 interact and cooperate in the transcriptional activation of the human immunodeficiency virus type 1 long terminal repeat in human microglial cells. J Biol Chem. 1997:272:31149-55.

42. Krebs FC, Goodenow MM, Wigdahl B. Neuroglial ATF/CREB factors interact with the human immunodeficiency virus type 1 long terminal repeat. J Neurovirol. 1997;3(Suppl 1):S28-32.

43. Li L, Aiamkitsumrit B, Pirrone V, Nonnemacher MR, Wojno A, Passic S, Flaig K, Kilareski E, Blakey B, Ku J, et al. Development of co-selected single nucleotide polymorphisms in the viral promoter precedes the onset of human immunodeficiency virus type 1-associated neurocognitive impairment. J Neurovirol. 2011;17:92-109.

44. Parikh N, Dampier W, Feng R, Passic SR, Zhong W, Frantz B, Blakey B, Aiamkitsumrit B, Pirrone V, Nonnemacher MR, et al. Cocaine alters cytokine profiles in HIV-1-infected African American individuals in the DrexelMed HIV/AIDS genetic analysis cohort. J Acquir Immune Defic Syndr. 2014;66:256-64.

45. Aiamkitsumrit B, Dampier W, Martin-Garcia J, Nonnemacher MR, Pirrone V, Ivanova T, Zhong W, Kilareski E, Aldigun H, Frantz B, et al. Defining differential genetic signatures in CXCR4- and the CCR5-utilizing HIV-1 Co-linear sequences. PLoS ONE. 2014;9:e107389.

46. Nonnemacher MR, Pirrone V, Feng R, Moldover B, Passic S, Aiamkitsumrit B, Dampier W, Wojno A, Kilareski E, Blakey B, et al. HIV-1 promoter single nucleotide polymorphisms are associated with clinical disease severity. PLoS One. 2016;11:e0150835.

47. Aimkitsumrit BD, Dampier W, Martin-Garcia J, Nonnemacher M, Pirrone V, Ivanova T, Zhong W, Kilareski E, Aldigun A, Frantz B, Rimbey M, Wojno A, Passic S, Williams J, Shah S, Blakely B, Parikh N, Jacobson J, Moldover B, Wigdahl B. Defining differential genetic signatures in CXCR4- and the CCR5-utilizing HIV-1 co-linear sequences. PLoS One. 2014;29:e107389.

48. Tamura K, Peterson D, Peterson N, Stecher G, Nei M, Kumar S. MEGA5: molecular evolutionary genetics analysis using maximum likelihood, evolutionary distance, and maximum parsimony methods. Mol Biol Evol. 2011;28:2731-9.

49. Schwartz GW, Hershberg U. Conserved variation: identifying patterns of stability and variability in BCR and TCR V genes with different diversity and richness metrics. Phys Biol. 2013;10:035005.

50. Ashkenazy H, Erez E, Martz E, Pupko T, Ben-Tal N. ConSurf 2010: calculating evolutionary conservation in sequence and structure of proteins and nucleic acids. Nucleic Acids Res. 2010;38:W529-33.

51. Yona G, Levitt M. Within the twilight zone: a sensitive profile-profile comparison tool based on information theory. J Mol Biol. 2002;315:1257-75.

52. Wang G, Dunbrack RL Jr. Scoring profile-to-profile sequence alignments. Protein Sci. 2004;13:1612-26.

53. Vacic V, lakoucheva LM, Radivojac P. Two Sample Logo: a graphical representation of the differences between two sets of sequence alignments. Bioinformatics. 2006;22:1536-7.

\section{Submit your next manuscript to BioMed Central and we will help you at every step:}

- We accept pre-submission inquiries

- Our selector tool helps you to find the most relevant journal

- We provide round the clock customer support

- Convenient online submission

- Thorough peer review

- Inclusion in PubMed and all major indexing services

- Maximum visibility for your research

Submit your manuscript at www.biomedcentral.com/submit
BioMed Central 